\title{
Evaluation of a Subset of the Solanum lycopersicum var. cerasiforme Core Collection for Horticultural Quality and Fruit Phenolic Content
}

\author{
Peter Boches, Brooke Peterschmidt, and James R. Myers ${ }^{1}$ \\ Department of Horticulture, Oregon State University, 4017 Ag \& Life Sciences \\ Building, Corvallis, OR 97330
}

Additional index words. flavonoid, phenylpropanoid, chlorogenic acid, rutin, naringenin, HPLC, Folin-Ciocalteau

\begin{abstract}
One hundred S. l. var. cerasiforme (Dunal) accessions from the 'Tanksley' designated core collection were evaluated for horticultural quality under greenhouse conditions. Fourteen selected accessions were grown under field conditions in a replicated trial to evaluate the fruit for phenolic content. Total fruit phenolics ranged from 44 to 82 $\mathrm{mg} / \mathbf{1 0 0} \mathrm{g}$ gallic acid equivalents (GAE) fresh weight (FW) as measured by Folin-Ciocalteau assay (F-C), and 12 to $108 \mathrm{mg} / 100 \mathrm{~g} \mathrm{FW}$ as measured by high-performance liquid chromatography (HPLC). Five accessions (LA1712, LA1455, LA2633, LA1668, and LA2632) had significantly higher total phenolics $(F-C)$ than cultivars $(P \leq 0.05)$. These five accessions also possessed interesting phenolics profiles, including high levels of caffeoylquinic acids (LA1620, LA1455, and LA2633) and rutin (LA2633).
\end{abstract}

Tomatoes are an important food crop and a significant constituent in the human diet, being the second most consumed vegetable in the United States after potatoes (Economic Research Service, USDA, 2008). Because they comprise a large portion of the average vegetable intake, tomatoes are an important source of phenolics in the human diet. Phenolics are a group of secondary plant metabolites that include phenolic acids, anthocyanins, and flavonoids. These compounds are produced only by plants and exhibit a broad range of health-promoting effects when consumed in foods of plant origin. Phenolics have been correlated with anti-inflammatory, antiviral, and antiallergenic effects (Kushad et al., 2003; Middleton and Kandaswami, 1993). Phenolics are also strong antioxidants, but recent research

Received for publication 17 June 2011. Accepted for publication 8 Sept. 2011.

Funding for this research was provided by grants from the Baggett-Frazier endowment and by a Howard-Hughes Medical Institute fellowship to Brooke Peterschmidt. This publication was made possible, in part, by the Mass Spectrometry Facilities and Services Core of the Environmental Health Sciences Center, Oregon State University, grant number P30 ES00210, National Institute of Environmental Health Sciences, National Institutes of Health.

We thank Steven Tanksley, Boris Igić, and Ingrid Phillips for providing access to the tomato germplasm and marker genotype data used in this work. Technical assistance with phenolics analysis was provided by Bob Durst, Fred Stevens, and Jeff Morre of Oregon State University.

These authors contributed equally to this article. This paper is a portion of theses submitted by P.B. and B.P. in partial fulfillment of degree requirements. ${ }^{1}$ To whom reprint requests should be addressed; e-mailmyersja@hort.oregonstate.edu. indicates that phenolics do not act as antioxidants in vivo (Lotito and Frei, 2006) suggesting their health benefits are derived from an unidentified mechanism.

Compared with many vegetables, tomato phenolic levels are relatively low, ranking 15 th in total phenolics of 23 vegetables (Vinson et al., 1998). The highest concentrations of these compounds are found in the skin of the fruit with lesser amounts found in the flesh (Kushad et al., 2003; Stewart et al., 2000). Considerable effort has been made to increase the levels of phenolics in tomato fruit through the use of genetic engineering (Schijlen et al., 2004, 2006) and introgression of genes from wild species (Willits et al., 2005). However, the use of genetic engineering is limited by public concerns over the safety of genetically modified foods, whereas the introgression of novel traits from wild species is often hindered by linkage of undesirable traits.

The feral or wild tomato subspecies Solanum lycopersicum var. cerasiforme ranges throughout much of South and Central America and represents the closest wild gene pool for the introgression of novel traits into cultivated tomato. Many $S$. $l$. var. cerasiforme accessions are similar in morphology to cultivated tomato; however, this subspecies has previously been reported to contain high levels of total phenolics (Hanson et al., 2004). Steve Tanksley designated a set of $100 \mathrm{~S} . l$. var. cerasiforme accessions as a core collection, which was assembled to maximize sampling of geographical and morphological diversity (S. Tanksley, Cornell University, personal communication). These accessions will be extensively genotyped using molecular markers and the information made publicly available (some marker information was already available by request). Using this core set of genotypes that has been previously characterized at the molecular level will facilitate introgression of these genes related to phenolic content into cultivars. The objective of this research was to evaluate tomato accessions for phenolic content with the aim of selecting accessions that could be crossed to elite lines for the development of high-phenolic tomato cultivars.

\section{Materials and Methods}

Plant material. One hundred accessions of $S$. $l$. var. cerasiforme were obtained from Steve Tanksley of Cornell University (personal communication). For the full list of accessions in the Tanksley core, see Boches (2009). Because some of these accessions originate from cross-pollinated, heterozygous populations, Tanksley proposed selfing them to homozygosity to provide a more permanent genetic resource amenable to genetic analysis. Seed was obtained from Tanksley in the S1 generation. All 100 accessions were evaluated under greenhouse conditions for horticultural qualities such as earliness and yield in the $\mathrm{S} 1$ generation. Greenhouse growth of tomato plants was as for Mes et al. (2008). In brief, tomato seeds were sown in Sunshine SB40 professional growing mix (Sun Gro Horticulture, Bellevue, WA) in 5-cm-diameter plastic transplant cells. Seedlings were transplanted into 3.8-L pots filled with Special Blend potting mix and $20 \mathrm{~g}$ of $14 \mathrm{~N}-6.1 \mathrm{P}-11.6 \mathrm{~K}$ slow-release fertilizer (Simplot 14-14-14) after 3 weeks. Plants were given $10 \mathrm{~g}$ of supplemental fertilizer after 4 weeks in the pots. Greenhouse temperature was set to $18{ }^{\circ} \mathrm{C}$ night and $25{ }^{\circ} \mathrm{C}$ day. Supplemental lighting was provided for $16 \mathrm{~h}$ per day by $400-\mathrm{W}$ metal halide and 400-W high-pressure sodium Sun System 3 high-intensity discharge lamps (Sunlight Supply, Woodland, WA). All accessions were evaluated for fruit and plant type.

Fourteen accessions with the best horticultural qualities in the group (earlier maturity, higher fruit yield and quality, compact plants) were grown at the OSU Vegetable Research Farm, Corvallis, OR, during the summer of 2007 in the S2 generation. 'Gold Nugget', 'Siletz', and LA1996, a high flavonoid introgression line that expresses the Anthocyanin fruit tomato (Aft) gene (Jones et al., 2003), were included as standards for low, medium, and high-phenolic tomato lines, respectively. Three replications of five plants each were arranged in a randomized complete block design. Plants were transplanted 21 May; field growth conditions were as described in Mes et al. (2008). Fruit samples for phenolic analysis (one $\approx 500$-g sample per plot) were harvested as fruit reached the red stage of maturity (as defined by Yamaguchi, 1983 ) in mid-August. Because samples were collected by weight, a variable number of fruit was collected per plot depending on the average fruit size; however, the sample size was based on a minimum of three fruit per plot. Based on the results of the field trial, the five accessions with the best horticultural qualities and the highest phenolic content 
(as measured by F-C; see Folin-Ciocalteau analysis subsequently) were grown under greenhouse conditions in the $\mathrm{S} 3$ generation.

Three replicates of one plant each were grown under the same greenhouse conditions as before and evaluated for phenolics content using HPLC. 'Legend', 'Gold Nugget', and LA1996 were used as standards in the second greenhouse evaluation. 'Legend' and 'Siletz' have very similar size and phenolic profiles and 'Legend' served as a substitute standard for 'Siletz'.

Samples and chemicals. Fruit were cut in half lengthwise, and seeds, placenta, and columella tissue were removed. The remaining pericarp and epidermal tissue were ground with liquid nitrogen to a fine powder using a mortar and pestle and weighed portions of this powder were used for extractions. For field samples, 70 to $150 \mathrm{mg}$ of the tomato powder was extracted in $300 \mu \mathrm{L}$ of acidified methanol $(1 \% \mathrm{HCl})$ overnight at $4{ }^{\circ} \mathrm{C}$ in $1.5 \mathrm{~mL}$ Eppendorf tubes. Extractions were brought to $500 \mu \mathrm{L}$ with nanopure $\mathrm{H}_{2} 0$, vortexed with 500 $\mu \mathrm{L}$ chloroform, and centrifuged at 13,000 rpm for $10 \mathrm{~min}$. The aqueous supernatant was removed and stored at $-20^{\circ} \mathrm{C}$ for further analysis. The greenhouse-grown samples were processed using a scaled-up version of this protocol $(1 \mathrm{~g}$ of powdered tissue was extracted in $5 \mathrm{~mL} \mathrm{60:40} \mathrm{1 \%} \mathrm{HCl} \mathrm{methanol:water} \mathrm{and} 5 \mathrm{~mL}$ chloroform in 15-mL glass tubes). Gallic acid, rutin, and 5-caffeoylquinic acid purchased from Sigma Chemical Corp (St. Louis, MO) were used as standards for F-C and HPLC.

Folin-Ciocalteau analysis. F-C assays were performed according to the method of Singleton and Rossi (1965) and Waterhouse (2006) with modifications. F-C reactions were performed on a microscale: each reaction contained $198 \mu \mathrm{L}$ water, $12.5 \mu \mathrm{L}$ F-C reagent (Sigma), $2.5 \mu \mathrm{L}$ of each sample or gallic acid standard, and $37.5 \mu \mathrm{L}$ sodium carbonate solution. Reactions were incubated for $1 \mathrm{~h}$ at room temperature, and absorbance at $765 \mathrm{~nm}$ was read using a SPECTRAmax microplate spectrophotometer (Molecular Devices, Sunnyvale, CA).

Liquid Chromatography-Diode Array Detection and Liquid Chromatography-Mass Spectroscopy analysis. HPLC analysis of greenhouse-grown fruit was performed on three samples from different plants. Columns and equipment used were as for Mes et al. (2008). The separation gradient and buffers were as described by Moco et al. (2006). A Hewlett-Packard (Palo Alto, CA) 1050 series autosampler and pump coupled to a HP $1040 \mathrm{M}$ diode array detector detection system was used. An ES Industries (West Berlin, NJ) reversed-phase LiChrospher RP18 end-capped column $(25 \mathrm{~cm} \times 4.6 \mathrm{~mm}, 5 \mu \mathrm{m}$ particle size $)$ was used with a guard column of the same material. The separation gradient and buffers followed that of the tomato metabolite database (Moco et al., 2006). Solvent A was formic acid:water $(1: 1000)$. Solvent $B$ was formic acid:acetonitrile $(1: 1000)$. The flow rate was $1 \mathrm{~mL} \cdot \mathrm{min}^{-1}$, and the injection volume was 40 $\mu \mathrm{L}$. The elution conditions were as follows: 0 to $45 \mathrm{~min}$, linear gradient from $5 \% \mathrm{~B}$ to $35 \% \mathrm{~B} ; 45$ to $50 \mathrm{~min}$, isocratic at $85 \% \mathrm{~B}$ to wash the column; and 50 to $60 \mathrm{~min}$, isocratic at $5 \% \mathrm{~B}$ to equilibrate the column. Peak spectra were monitored at $280 \mathrm{~nm}$. Ultraviolet spectra were recorded from 200 to $600 \mathrm{~nm}$ in $0.5-\mathrm{nm}$ intervals. HPLC data were analyzed using Agilent ChemStation Rev.A.09.01 (Santa Clara, CA). Compounds were quantified as equivalents to chlorogenic acid or rutin using a standard curve and summarized according to compound classes. Standards were used in the range of 25 to $250 \mathrm{mg} \cdot \mathrm{L}^{-1}$ for rutin and 25 to $500 \mathrm{mg} \cdot \mathrm{L}^{-1}$ for chlorogenic acid. Liquid chromatography-mass spectroscopy (LC-MS) was performed using the same column, buffers, and elution conditions on a Shimadzu LC20-AD chromatography system (Shimadzu,
Columbia, MD) coupled to an ABI 3200 QTRAP LC/MS/MS (Applied Biosystems, Foster City, CA) triple quadrupole mass spectrometer equipped with a Turbo $\mathrm{V}$ ionization source.

Statistical analysis. SAS software (Cary, NC) was used for analysis of variance and means separations using Fisher's least significant difference.

\section{Results}

A wide range of fruit and plant types were observed among $S$. $l$. var. cerasiforme accessions. The vast majority of $S$. $l$. var. cerasiforme accessions evaluated had extremely late maturity (greater than $120 \mathrm{~d}$ ) or low yield (measured as the quantity of fruit relative to vegetation) relative to cultivated tomatoes Because tomato is a facultative short-day plant (Binchy and Morgan, 1970) that flowers best under a photoperiod of $12 \mathrm{~h}$ or less, the growth conditions provided ( $16 \mathrm{~h}$ of supplemental lighting) may have inhibited flowering in some accessions. Other undesirable qualities for our objectives included inflorescences which resumed vegetative growth, mealy fruit, seedy fruit, thick-skinned fruit, pale fruit color, and larger fruit (as a result of the inverse correlation between fruit size and phenolic content). Accessions were evaluated for these characters and the 14 best accessions based on a combination of these criteria were selected for further evaluation in a replicated field trial. In many cases, early-maturing accessions were low-yielding, or high-yielding accessions had poor fruit quality, so the final decision was made subjectively.

The 14 selected accessions were evaluated further for horticultural quality (including flavor) in the field trial (Table 1). Fungal diseases including powdery mildew (Oidium lycopersicum) and gray mold (Botrytis cinerea) were a problem in some accessions and strong

Table 1. Phenolic content of fruit extracts, average fruit weights, and field notes for S. $l$. var. cerasiforme accessions and cultivated tomatoes from 2007 field trial. ${ }^{z}$

\begin{tabular}{|c|c|c|c|c|c|}
\hline $\begin{array}{l}\text { Entry } \\
\end{array}$ & Mean GAE & & $\mathrm{SE}$ & Field notes & Avg fruit wt (g) \\
\hline LA1455 & $75.5_{\mathrm{ab}}^{\mathrm{a}}$ & \pm & 8.7 & $\begin{array}{l}\text { Sweet fruit; upright growth habit; thick skin; good yield and yield architecture; } \\
\text { flavor ok; fruit with glandular trichomes }\end{array}$ & 3.3 \\
\hline LA2633 & $73.0_{\mathrm{abc}}$ & \pm & 3.3 & Hard skin, very sweet, high solids; later maturity, good yield, long fruit trusses & 2.3 \\
\hline LA2632 & 67.5 abcde & \pm & 4.4 & Very small pubescent fruit; poor flavor (bland); firm pericarp & 2.1 \\
\hline LA1620 & 63.6 abcdef & \pm & 6.5 & $\begin{array}{l}\text { Good yield and fungal disease resistance; relatively good fruit quality, excessive } \\
\text { gel on seeds, earlier than LA1549 }\end{array}$ & 5.9 \\
\hline LA1338 & 57.2 bcdefg & \pm & 17.3 & $\begin{array}{l}\text { Early, very dark red fruit; very poor fungal disease resistance; horrible flavor } \\
\text { (possibly resulting from fungal infection?) }\end{array}$ & 7.1 \\
\hline LA2626 & $51.9 \mathrm{cdefg}$ & \pm & 7.2 & $\begin{array}{l}\text { Bad taste (astringent or causing a prickly sensation in throat); poor fungal disease } \\
\text { resistance; good yield, early maturing }\end{array}$ & 6.0 \\
\hline LA1290 & $45.4_{\text {defg }}$ & \pm & 0.8 & Larger fruit, watery flavor; good yield; variable fruit shape & 14.2 \\
\hline LA2308 & $44.2 \mathrm{efg}$ & \pm & 1.7 & Large fruit; medium to good yield; bitter flavor, very astringent & 23.6 \\
\hline
\end{tabular}

${ }^{2}$ Phenolics content of fruit extracts was measured by Folin-Ciocalteau assay and expressed as gallic acid equivalents (GAE). Means followed by the same letter are not significantly different as measured by Fisher's least significant difference $(P \leq 0.05)$. Fruit weights are the average of 10 ripe fruit. 
genotype-dependent responses were observed. There was no correlation between phenolic content of the fruit and fungal disease resistance (Table 1). Some of the best accessions in terms of horticultural quality (for a cherry tomato) were LA1620, LA1549, LA1668, LA1512, LA2633, and LA1455.

Total phenolic content of fruit extracts from field-grown material are presented in Table 1. Five accessions (LA1455, LA1668, LA1712, LA2632, and LA2633) had significantly higher fruit phenolics than cultivars as measured by the F-C assay (total phenolics F-C) (Table 1). Only LA1455, LA1712, and LA2633 had significantly higher total phenolics than LA1996 (Table 1). There was a modest but statistically significant negative correlation between total phenolics (F-C) and fruit weight (Pearson's correlation coefficient $=-0.49, P=$ 0.046 ). Five accessions were selected for further evaluation in the greenhouse (LA1455, LA1549, LA1620, LA1712, and LA2633). The decision of which accessions to use for further evaluation was based on a combination of their F-C total phenolic scores and their horticultural quality (Table 1).

HPLC-photodiode array (PDA) information for greenhouse-grown material (Fig. 1) was based on three replicates (each replicate consisted of ripe fruit tissue from an individual greenhouse-grown plant). LA2633 was notable for having levels of quercetin and kaempferol derivatives that were significantly higher than cultivars and many other $S$. l. var. cerasforme accessions. Most $S$. $l$. var. cerasforme accessions had significantly higher levels of mono, di-, and triacylquinic acids than cultivars.

Peak assignments (Figs. 2 and 3; Table 2) were made using a combination of peak retention times, ultraviolet-spectra, and mass spectra. Mass spectra and ultraviolet-spectra were acquired on separate instruments. Mass spectra were only acquired for the genotypes Legend and LA2633. Retention times for major tomato phenolic compounds (e.g., chlorogenic acid, rutin, and naringenin chalcone) were $\approx 2$ min later on the HPLC-MS system than on the HPLC-PDA system, but the chromatograms could still be aligned fairly well (Figs. 2 and 3). Twelve peaks were positively identified using a combination of ultravioletand accurate mass spectra (Table 2). HPLCPDA chromatograms (Fig. 3) revealed that $S$. $l$. var. cerasforme accessions contained numerous compounds that were not detected or detected in only trace quantities from cultivars (e.g., Peaks 8,9 , and 10). Phenolic content was summarized by compound class (caffeoylquinic acids, Peak 2; diacylquinic acids, Peak 9; quercetin derivatives, Peaks 3 and 6; rutin, Peak 7) and quantitated using a standard curve from chlorogenic acid (caffeoylquinic acids) or rutin (quercetin derivatives) based on peak identifications in Table 2 and/or peak assignments to compound class (Fig. 1). Standard curves were linear over the range sampled $\left(R^{2} \geq 0.9816\right)$.

\section{Discussion}

Tomato is a model species for the study of fruit development and there has been a recent
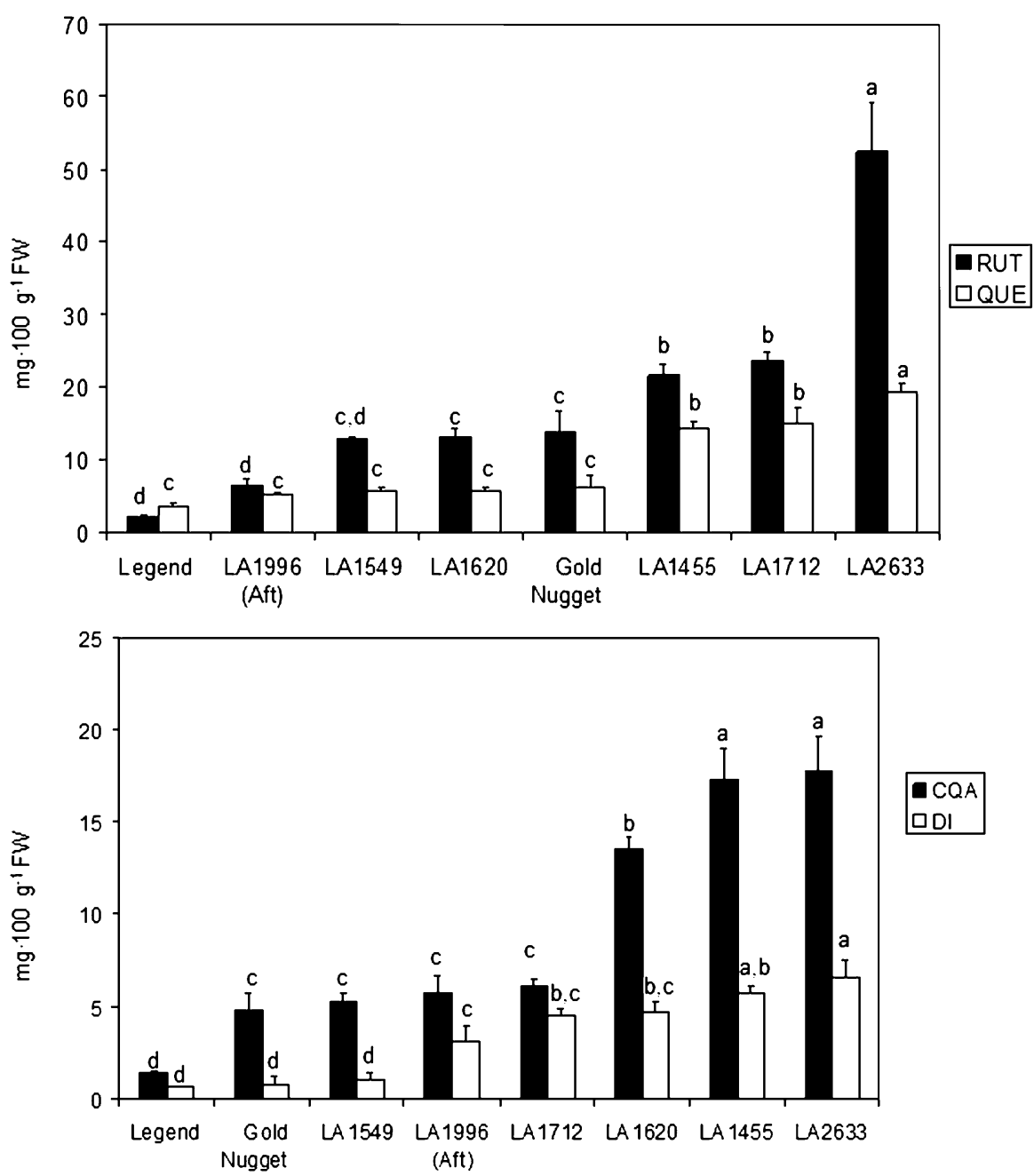

Fig. 1. Phenolic content (mg/100 g fresh weight, measured by high-performance liquid chromatographyphotodiode array) of $S$. $l$. var. cerasiforme accessions. Values are the mean of three biological replicates; error bars $=1 \mathrm{SE}$. Means sharing a letter (within a series of phenolic compounds) are not significantly different, Fisher's least significant difference, $P \leq 0.05$. Series abbreviations: RUT = rutin; $\mathrm{QUE}=$ quercetin derivative other than rutin; CQA $=$ caffeoylquinic acid isomers; $\mathrm{DI}=$ dicaffeoylquinic acid isomers.

explosion in metabolomics research related to analysis of phenolic compounds in tomato (Iijima et al., 2008; Mintz-Oron et al., 2008; Moco et al., 2006, 2007). Many compounds present only in trace amounts in cultivated tomato were present at higher levels in the $S$. l. var. cerasiforme accessions evaluated here, including quercetin 3-2" apiosyl-6" rhamnosylglucoside (Butelli et al., 2008; Slimestad et al., 2008), a quercetin dihexose-deoxyhexose (Iijima et al., 2008; Moco et al., 2007), feruloylquinic acid (Moco et al., 2006), dicaffeoylquinic acids (Luo et al., 2008; Moco et al., 2006), and tricaffeoylquinic acids (Luo et al., 2008; Moco et al., 2006). We also found two putative isomers of feruloylquinic acid $\left([\mathrm{M}-\mathrm{H}]^{-}=367.0\right)$ in which only one has been previously reported in tomato (Moco et al., 2006).

Many of the $100 \mathrm{~S}$. l. var. cerasiforme accessions that were evaluated in the greenhouse had very late maturity (over $120 \mathrm{~d}$ ) or low yields resulting from excessive vegetative growth and were not evaluated for phenolic content in the field or otherwise. Field evaluation revealed that some accessions suffered from susceptibility to fungal diseases, poor flavor, or other defects (Table 1). LA1712, LA1455, LA2633, and LA1549 were accessions with better horticultural quality and higher phenolic content over multiple environments. There was no correlation between resistance to fungal diseases and fruit phenolic content (Table 1). However, leaf phenolic content might be expected to play a more important role in influencing disease resistance. Leaf phenolic content of the 14 accessions in the field trial was measured by F-C and ranged from 322 GAE to 979 GAE but showed poor correlation with levels of fungal disease resistance (data not shown).

HPLC-PDA chromatograms of these accessions revealed important differences in their phenolic profiles (Fig. 2). LA1712 (from Pejibaye, Costa Rica) was notable for having large amounts of di- and tricaffeoylquinic acids (Peaks 9 and 10). LA1712 possessed large amounts of a phenolic compound that was not positively identified (the large peak in between Peaks 7 and 9). This peak had a retention time of 32.44 min and an ultraviolet max $_{\text {max }}$ 

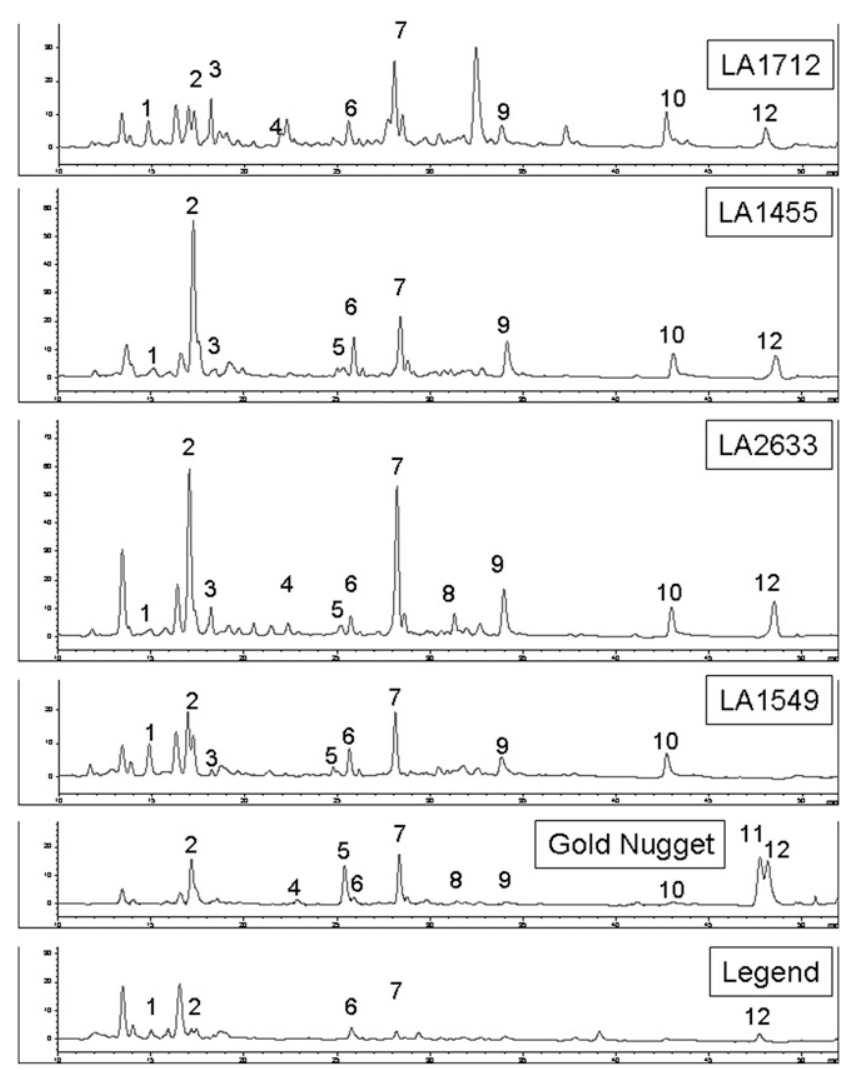

Fig. 2. High-performance liquid chromatography-photodiode array chromatograms $(280 \mathrm{~nm})$ of selected accessions in this study from greenhouse-grown fruit. For peak assignments, see Table 2. All chromatograms to the same scale.
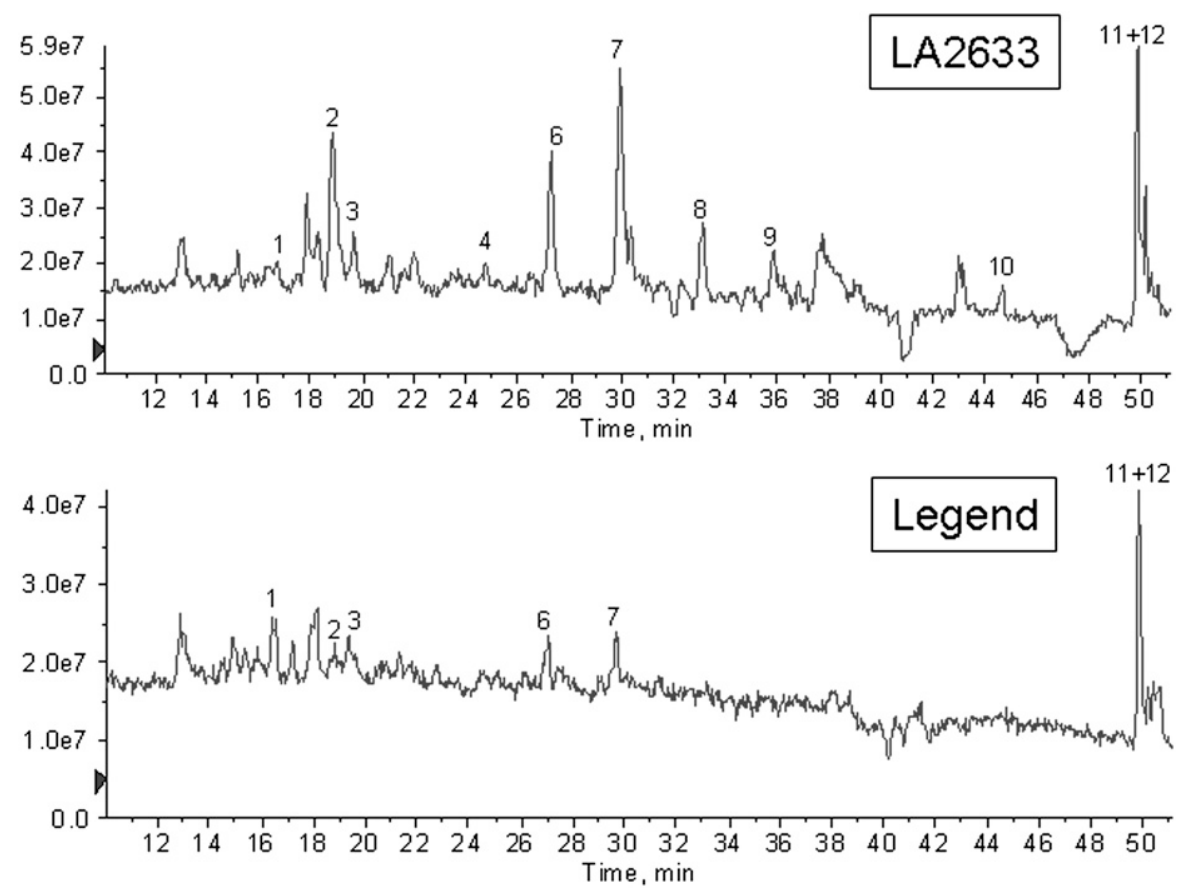

Fig. 3. Total ion chromatograms of LA2633 and 'Legend' from liquid chromatography-mass spectroscopy analysis. Peak assignments are as for Figure 2 and Table 2.

of $284 \mathrm{~nm}$ with a shoulder at $326 \mathrm{~nm}$, a spectrum that could be consistent with a chalcone derivative or a coumaric acid derivative. This compound was one of the predominant phenolics in LA1712 but was present in only trace amounts in other accessions. Because mass spectra were only obtained for the genotypes LA2633 and 'Legend', mass spectra for this peak were not available. Because this peak was not positively identified, it was not assigned a peak number (Figs. 2 and 3; Table 2). LA1712 had poor fungal disease resistance and very acidic flavor, however. LA1455 (from Nuevo Leon, Mexico) had very high levels of 4-caffeoylquinic acid (chlorogenic acid). LA1455 also had good (upright) plant architecture and yield and small, sweet, glandular fruit. LA2633 (from Cusco, Peru) had high levels of chlorogenic acid (Peak 2), kaempferol and quercetin derivatives (Peaks $3,6,7$, and 8), and di- and triacylquinic acids (Peaks 9 and 10). LA2633 had hard skin, sweet fruit with high solids, good yield, and long fruit trusses. LA1549 (from Pasco, Peru) had moderately high levels of chlorogenic acid (Peak 2), quercetin derivatives (Peaks 3, 6 , and 7), and di- and tricaffeoylquinic acids (Peaks 9 and 10).

The levels of phenolic compounds found in tomatoes or other fruits vary greatly depending on the sample preparation method, tissue(s) sampled, genotype, and growing conditions (Robbins, 2003). Among cultivated tomatoes, tissue type and maturity stage have major effects on the quantity and profile of compounds (Moco et al., 2007). According to the USDA Database for the Flavonoid Content of Selected Foods, Release 2.1 (United States Department of Agriculture, 2007), the yearround average naringenin and quercetin content of red tomatoes is 0.68 and $0.58 \mathrm{mg} / 100 \mathrm{~g}$ FW, respectively; whereas for cherry tomatoes, these values are 3.19 and $2.76 \mathrm{mg} / 100 \mathrm{~g}$ $\mathrm{FW}$, respectively. These values are based on the entire edible portion of the fruit. In this study, seeds, gel, and columellar tissue were excluded because the inclusion of liquid nitrogen ground seeds in the extraction might affect the fruit phenolic profile in ways that are biologically unrealistic as far as human consumption is concerned. Another reason for excluding these tissues was to avoid the dilution of minor compounds below the limit of detection without concentration of the samples, which can cause degradation. We report values four to eight times those of the USDA for cultivated tomatoes in the tissue sampled here (pericarp and epidermis). This value is reasonable given that the seeds, columella, gel, and placental tissues constitute a significant portion of the weight of a fresh tomato and the phenolics are concentrated in the outer layers of the pericarp and epidermis. Recently, flavonoid concentrations of up $200 \mathrm{mg} / 100 \mathrm{~g}$ FW have been reported for tomato peel (Giuntini et al., 2008; Torres et al., 2005). Thus, we believe that the high concentration of quercetin and other phenolics reported here for some smallfruited, wild accessions (up to $70 \mathrm{mg} / 100 \mathrm{~g}$ FW quercetin in LA2633 under greenhouse conditions) is relatively accurate.

In general, $S . l$. var. cerasiforme accessions did not have particularly high levels of naringenin (Peak 11) or naringenin chalcone (Peak 12), which have sometimes been reported to be the predominant flavonoids in tomato. In fact, the highest levels of these compounds were found in the cultivar Gold Nugget in our study (Fig. 2). We mainly observed naringenin chalcone rather than naringenin. This is in agreement with the observation of Slimestad 
Table 2. Metabolites identified by high-performance liquid chromatography-photodiode array (HPLC-PDA) and liquid chromatography-mass spectroscopy. ${ }^{z}$

\begin{tabular}{|c|c|c|c|c|c|}
\hline Peak & RT & Mass & ID & Ultraviolet-Vis & Reference \\
\hline 1 & 15.17 & 341.0 & Caffeic acid hexose & $328,298 \mathrm{sh}$ & Moco et al., 2007 \\
\hline 3 & 18.25 & 771.3 & $\begin{array}{l}\text { Quercetin dihexose-deoxyhexose } \\
\text { (quercetin 3-o-rutinoside-7-o-glucoside) }\end{array}$ & 256,352 & Iijima et al., 2008; Moco et al., 2007 \\
\hline 4 & 21.48 & 367.0 & Feruloylquinic acid isomer & $328,298 \mathrm{sh}$ & $\begin{array}{l}\text { Clifford et al., 2003; Kammerer et al., 2004; } \\
\quad \text { Moco et al., } 2006\end{array}$ \\
\hline 5 & 25.23 & 367.0 & Feruloylquinic acid isomer & 326 & Clifford et al., 2003; Moco et al., 2006 \\
\hline 7 & 28.23 & 609.2 & Rutin & 256,354 & Slimestad et al., 2008; many others \\
\hline 8 & 31.31 & 593.2 & Kaempferol 3-6" rhamnosylglucoside & 264,344 & $\begin{array}{l}\text { Iijima et al., 2008; Moco et al., 2007; } \\
\text { Slimestad et al., } 2008\end{array}$ \\
\hline 9 & 33.98 & 515.3 & Dicaffeoylquinic acid & $326,298 \mathrm{sh}$ & Clifford et al., 2003; Moco et al., 2006 \\
\hline 10 & 42.99 & 677.3 & Tricaffeoylquinic acid & $330,294 \mathrm{sh}$ & Clifford et al., 2003; Moco et al., 2006 \\
\hline 11 & 47.74 & NA & Naringenin & 288,344 sh & Slimestad et al., 2008; many others \\
\hline 12 & 48.5 & 271.0 & Naringenin chalcone & 366 & Slimestad et al., 2008; many others \\
\hline
\end{tabular}

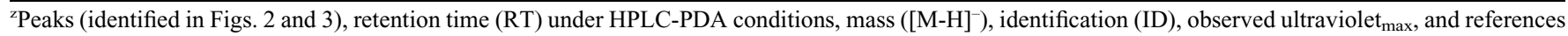
from the literature.

$\mathrm{NA}=$ not available; $\mathrm{sh}=$ shoulder of ultraviolet $_{\max }$.

et al. (2008) that naringenin chalcone is the native compound. According to Slimestad et al. (2008), reports of naringenin as the predominant compound are the result of the spontaneous isomerization of naringenin chalcone into naringenin. The two compounds have very different ultraviolet maxima, and because standards for naringenin chalcone were not available to us, we did not estimate naringenin chalcone levels.

Hanson et al. (2004) reported total phenolics as measured by the F-C method in tomato in the range of 60 to $90 \mathrm{mg} / 100 \mathrm{~g} \mathrm{FW}$ for cultivars and $150 \mathrm{mg} / 100 \mathrm{~g} \mathrm{FW}$ for small-fruited $S$. pimpinellifolium accessions. Hernández et al. (2007) reported lower values in the range of 10 to $30 \mathrm{mg} / 100 \mathrm{~g} \mathrm{FW}$ for cultivated tomatoes. These differences could be the result of the sample preparation method (fresh versus frozen puree, respectively), but differences in the standard curves are another possible explanation. We report values intermediate between the two studies (40 mg/100 g FW).

Smaller tomato fruit also have higher phenolic concentrations as a result of their increased surface area:volume ratio. There were several indications that the high phenolic content observed in $S$. l. var. cerasiforme accessions was not the result of small fruit size alone, however. We found a significant correlation between fruit size and phenolics content, but fruit size was not strictly correlated with phenolic content, especially among accessions of $S$. l. var. cerasiforme (e.g., LA1512 had small fruit but only modest phenolic levels).

Several $S$. $l$. var. cerasiforme accessions had levels of di- and triacylquinic acids that were significantly higher than cultivated tomatoes (Fig. 1), making the $S$. $l$. var. cerasiforme accessions evaluated here a good source for this trait. MicroTom cultivars transformed with $A t \mathrm{MYB} 12$ produced di- and triacylquinic acids at a concentration of 4 to $6 \mathrm{mg} \cdot \mathrm{g}^{-1}$ dry weight (Luo et al., 2008), a level similar to that observed for high-phenolic $S$. $l$. var. cerasiforme accessions in this study (4 to 7 $\mathrm{mg} / 100 \mathrm{~g} \mathrm{FW}$ ). The genetic basis of this trait therefore seems more likely to be the result of a difference in regulation of the pathway leading to these compounds rather than a lack of the necessary structural genes in the cultivated tomato.

This study provides the basis for a more extensive candidate gene analysis using classical mutants, mapped expressed sequence tags, and the tomato genome sequence. Because the Tanksley core set has been genotyped extensively, this information could be to facilitate introgression of high phenolic traits. S3 plants of LA1455, LA1549, LA1620, LA1712, and LA2633 were crossed to high anthocyanin (AftAftlatvatv) and high flavonol (AftAft/atvatv/awaw) tomato lines with the objective of developing tomato lines that have elevated fruit phenolic levels or unique phenolic profiles. Some of these populations could also be used for mapping genes related to the phenolic biosynthetic pathway. LA2633 exhibits a high degree of DNA marker polymorphism (Boches, 2009) as well as elevated levels of a wide range of polyphenolics, making it an ideal candidate for developing mapping populations. In contrast, $S$. $l$. var. cerasiforme accessions with high phenolics and a lower proportion of wild species alleles such as LA1712 (Boches, 2009) might make better candidates for the introducing the trait into cultivars to reduce linkage drag to unfavorable alleles for other traits.

In conclusion, screening of a genotypically characterized $S$. $l$. var. cerasiforme core collection for accessions with better horticultural quality and high phenolic content identified several accessions with elevated fruit phenolic content and unique fruit phenolic profiles. These accessions represent a useful resource for developing high-phenolic tomato germplasm or identifying genes associated with phenylpropanoid metabolism.

\section{Literature Cited}

Binchy, A. and J.V. Morgan. 1970. Influence of light intensity and photoperiod on inflorescence initiation in tomatoes. Ir. J. Agr. Res. 9:261269.

Boches, P.S. 2009. Breeding tomato for increased fruit phenolics. PhD thesis, Oregon State University.
10 Mar. 2011. <http://hdl.handle.net/1957/ 11136>.

Butelli, E., L. Titta, M. Giorgio, H.P. Mock, A. Matros, S. Peterek, E.G.W.M. Schijlen, R.D. Hall, A.G. Bovy, J. Luo, and C. Martin. 2008. Enrichment of tomato fruit with health-promoting anthocyanins by expression of select transcription factors. Nat. Biotechnol. 26:1301-1308.

Clifford, M.N., K.L. Johnston, S. Knight, and N. Kuhnert. 2003. A hierarchical scheme for LC$\mathrm{MS}^{n}$ identification of chlorogenic acids. J. Agr. Food Chem. 51:2900-2911.

Economic Research Service,USDA. 2008. Vegetables and melons situation and outlook yearbook. VGS2008. 30 Oct. 2008. <http://www.ers.usda.gov/ Publications/VGS/2008/05May/VGS2008.pdfs.

Giuntini, D., V. Lazzeri, V. Calvenzani, C. Dall'Asta, G. Galaverna, C. Tonelli, K. Petroni, and A. Ranieri. 2008. Flavonoid profiling and biosynthetic gene expression in flesh and peel of two tomato genotypes grown under UV-Bdepleted conditions during ripening. J. Agr. Food Chem. 56:5905-5915.

Hanson, P.M., R. Yang, J. Wu, J. Chen, D. Ledesma, S.C.S. Tsou, and T.C. Lee. 2004. Variation for antioxidant activity and antioxidants in tomato. J. Amer. Soc. Hort. Sci. 129:704-711.

Hernández, M., E. Rodríguez, and C. Díaz. 2007. Free hydroxycinnamic acids, lycopene, and color parameters in tomato cultivars. J. Agr. Food Chem. 55:8604-8616.

Iijima, Y., Y. Nakamura, Y. Ogata, K. Tanaka, S. Sakurai, K. Suda, T. Suzuki, H. Suzuki, K. Okazaki, M. Kitayama, S. Kanaya, K. Aoki, and D. Shibata. 2008. Metabolite annotations based on the integration of mass spectral information. Plant J. 54:949-962.

Jones, C.M., P. Mes, and J.R. Myers. 2003. Characterization and identification of the anthocyanin fruit (Aft) tomato. J. Hered. 94:449-456.

Kammerer, D., R. Carle, and A. Schieber. 2004 Characterization of phenolic acids in black carrots (Daucus carota ssp. Sativus var. atrorubens Alef.) by high-performance liquid chromatography/electrospray ionization mass spectrometry. Rapid Commun. Mass Spectrom. 18:1331-1340.

Kushad, M.M., J. Masiunas, M.A.L. Smith, W. Kalt, and K. Eastman. 2003. Health promoting phytochemicals in vegetables. Hort. Rev. 28:125-185.

Lotito, S.B. and B. Frei. 2006. Consumption of flavonoid-rich foods and increased plasma antioxidant capacity in humans: Cause, consequence, or epiphenomenon? Free Radic. Biol. Med. 46:1727-1746. 
Luo, J., E. Butelli, L. Hill, A. Parr, R. Nigeweg, P. Bailey, B. Weisshaar, and C. Martin. 2008. AtMYB12 regulates caffeoyl quinic acid and flavonol synthesis in tomato: Expression in fruit results in very high levels of both types of polyphenol. Plant J. 56:316-326.

Mes, P.J., P. Boches, J.R. Myers, and R. Durst. 2008. Characterization of tomatoes expressing anthocyanin in the fruit. J. Amer. Soc. Hort. Sci. 133:262-269.

Middleton, E. and C. Kandaswami. 1993. The impact of flavonoids on mammalian biology: Implications for immunity, inflammation and cancer, p. 619-644. In: Harborne, J.B. (ed.). The flavonoids: Advances in research since 1986. Chapman and Hall, London, UK.

Mintz-Oron, S., T. Mandel, I. Rogachev, L. Feldberg, O. Lotan, M. Yativ, Z. Wang, R. Jetter, I. Venger, A. Adato, and A. Aharoni. 2008. Gene expression and metabolism in tomato fruit surfaces. Plant Physiol. 147:823-851.

Moco, S., R.J. Bino, O. Vorst, H.A. Verhoeven, J. de Groot, T.A. van Beek, J. Vervoort, and C.H.R. de Vos. 2006. A liquid chromatographymass spectrometry-based metabolome database for tomato. Plant Physiol. 141:1205-1218.

Moco, S., E. Capanoglu, Y. Tikunov, R.J. Bino, D. Boyacioglu, R.D. Hall, J. Vervoort, and R.C.H.
De Vos. 2007. Tissue specialization at the metabolite level is perceived during the development of tomato fruit. J. Expt. Bot. 58:41314146.

Robbins, R. 2003. Phenolic acids in foods: An overview of analytical methodology. J. Agr. Food Chem. 51:2866-2887.

Schijlen, E., C.H. Ric de Vos, H. Jonker, H. van den Broeck, J. Molthoff, A. van Tunen, S. Martens, and A. Bovy. 2006. Pathway engineering for phytochemicals leading to the production of novel flavonoids in tomato fruit. Plant Biotechnol. J. 4:433-444.

Schijlen, E.G.W.M., C.H. Ric de Vos, A.J. van Tunen, and A.G. Bovy. 2004. Modification of flavonoid biosynthesis in crop plants. Phytochemistry 65:2631-2648.

Singleton, V.L. and J.A. Rossi. 1965. Colorimetry of total phenolics with phospomolybdicphosphotungstic acid reagents. Amer. J. Enol. Viticult. 16:144-158.

Slimestad, R., T. Fossen, and M.J. Verheul. 2008. The flavonoids of tomatoes. J. Agr. Food Chem. 56:2436-2442.

Stewart, A.J., S. Bozonnet, W. Mullen, G.I. Jenkins, M.E.J. Lean, and A. Crozier. 2000. Occurrence of flavonols in tomatoes and tomato-based products. J. Agr. Food Chem. 48:2663-2669.
Torres, C.A., N.M. Davies, J.A. Yanez, and P.K. Andrews. 2005. Disposition of selected flavonoids in fruit tissues of various tomato (Lycopersicon esculentum Mill.) genotypes. J. Agr. Food Chem. 53:9536-9543.

United States Department of Agriculture. 2007. USDA Database for the Flavonoid Content of Selected Foods, Release 2.1. 26 Jan. 2009. < http:// www.nal.usda.gov/fnic/foodcomp/Data/Flav/ Flav02-1.pdf>.

Vinson, J.A., Y. Hao, X. Su, and L. Zubik. 1998. Phenol antioxidant quantity and quality in foods. Vegetables. J. Agr. Food Chem. 46:3630 3634 .

Waterhouse, A. 2006. Folin-Ciocalteau micro method for total phenol in wine. Dept. of Viticulture and Enology, University of California, Davis. 1 Nov. 2008. <http://waterhouse.ucdavis edu/phenol/folinmicro.htm $>$.

Willits, M.G., C.M. Kramer, R.T.N. Prata, V. De Luca, B.G. Potter, J.C. Steffens, and G. Graser. 2005. Utilization of the genetic resources of wild species to create a nontransgenic high flavonoid tomato. J. Agr. Food Chem. 53:1231-1236.

Yamaguchi, M. 1983. Solanaceous fruits: Tomato, eggplant, peppers, and others, p. 291-312. In: Yamaguchi, M. (ed.). World vegetables. AVI Books, New York, NY. 